\title{
Habilidades cognitivas y funciones neuropsicológicas ejecutivas en niños bilingües (lengua nativa-castellano) y monolingües castellano
}

\section{Cognitive skills and executive neuropsychological functions in bilingual children (native language-castilian) and monolinguals castilian}

\author{
Ricardo Canales Gabriel ${ }^{1 *}$ \\ Esther Velarde Consoli ${ }^{2}$ \\ Juan Ramírez Mendoza ${ }^{3}$
}

Universidad Nacional Mayor de San Marcos

\author{
Susana Lingán Huamán ${ }^{4}$ \\ Universidad San Ignacio de Loyola
}

Recibido: $08-10-17$

Aceptado: $21-11-17$

\section{Resumen}

El estudio buscó establecer si existen diferencias en los niveles de desarrollo de habilidades cognitivas y funciones ejecutivas en niños bilingües y niños monolingües castellano, pertenecientes al mismo nivel socio-económico. Se examinó a 20 alumnos de $5^{\circ}$ y $6^{\circ}$ grado de la Comunidad de Socos; 24 alumnos de $5^{\circ}$ y $6^{\circ}$ grado de la Institución educativa "Virgen del Carmen", Pilacucho, Huamanga; 24 estudiantes de la I.E. intercultural bilingüe: "Comunidad Shipiba" en Cantagallo, Lima; y 18 estudiantes de $5^{\circ}$ y $6^{\circ}$ grado de "Santa Rosa de América" del Callao; con edades que fluctúan entre los 10 y 12 años. Se examinó inteligencia con el Test de Raven y funciones neuropsicológicas ejecutivas con algunos sub tests del BANFE (Flores-Ostrosky, 2012). Los resultados mostraron diferencias significativas entre los grupos, tanto en funcionamiento cognitivo como en las funciones ejecutivas.

Los alumnos que rindieron más bajo en ambas variables, fueron los niños de Socos, Ayacucho, zona rural y quechua-hablante. Luego se ubicaron los alumnos de Huamanga, capital del departamento de Ayacucho. Los que rindieron mejor fueron los alumnos del Callao y los niños de la comunidad shipiba de Cantagallo-Lima, con influencia de otro tipo de bilinguismo. Se aprecia que los factores disortogénicos: pobreza extrema, marginación social, el problema del bilinguismo quechua-castellano, estarían relacionados con el desarrollo cognitivo y de funciones ejecutivas en los niños. En conclusión se encontró diferencias signifícativas entre los grupos de estudiantes de diferentes realidades socio-culturales a nivel de funciones neuropsicológicas ejecutivas y en habilidades cognitivas.

Palabras clave: Bilingüismo; funciones neuropsicológicas ejecutivas; realidad socio-cultural.

\footnotetext{
1 Universidad Nacional Mayor de San Marcos, Facultad de Psicología.

* Correspondencia Email: rcanalesg@unmsm.edu.pe

2 Universidad Nacional Mayor de San Marcos, Facultad de Educación. Email: emvelarde@hotmail.com 3 Universidad Nacional Mayor de San Marcos, Facultad de Psicología. Email:r.juan19_94@hotmail.com 4 Universidad San Ignacio de Loyola. Email: ksusanalingan39@gmail.com
}

(C) Los autores. Este artículo es publicado por la Revista de Investigación en Psicología de la Facultad de Psicología, Universidad Nacional Mayor de San Marcos. Este es un artículo de acceso abierto, distribuido bajo los términos de la licencia Creative Commons Atribucion - No Comercia_Compartir Igual 4.0 Internacional. (http://creativecommons.org/licenses/by-nc-sa/4.0/) que permite el uso no comercial, distribución y reproducción en cualquier medio, siempre que la obra original sea debidamente citada. 


\begin{abstract}
The study sought to establish whether there are differences in levels of cognitive skills development and executive functions in bilingual children and Spanish monolingual children, belonging to the same socio-economic level. Twenty students from 5th and 6th grades of the Community of Socos were examined; 24 students of 5 th and 6th grade of the educational institution "Virgen del Carmen", Pilacucho, Huamanga; 24 students from the I.E. intercultural bilingual: "Shipiba Community" in Cantagallo, Lima; and 18 students of $5^{\circ}$ and $6^{\circ}$ degree of "Santa Rosa de America" of Callao; with ages ranging from 10 to 12 years.

They were examined intelligence with the Raven Test and executive neuropsychological functions with some subtests of BANFE (Flores-Ostrosky, 2012). The results showed significant differences between the groups, both in cognitive functioning and executive functions. The students who yielded the lowest in both variables were the children of Socos, Ayacucho, rural and Quechua-speaking. Then the students of Huamanga, capital of the department of Ayacucho. The best performers were students from Callao and children from the Shipiba community of Cantagallo-Lima, influenced by other types of bilingualism.

It is noted that dysortogenic factors: extreme poverty, social marginalization, and the problem of Quechua-Castilian bilingualism, would be related to cognitive development and executive functions in children. In conclusion, we found significant differences between groups of students from different socio-cultural realities at the level of executive neuropsychological functions and cognitive abilities.
\end{abstract}

Keywords: Bilingualism; executive neuropsychological functions; sociocultural reality.

Partimos de la constatación de lo compleja y diversa que es la realidad socio-cultural en que crecen nuestros niños en el Perú. González (2006), remarcó la presencia de los factores disortogénicos en el desarrollo psicológico del niño peruano. Es decir, como la pobreza extrema, la marginalidad social, el analfabetismo de los padres, los problemas de bilinguismo quechua-castellano, etc. influyen negativamente en dicho desarrollo.

En particular, sobre el Bilinguismo en el Perú, González (2006) realizó un estudio pionero en Ayacucho sobre las relaciones entre bilingüismo y desarrollo del pensamiento operatorio. Escogió una muestra de 157 niños de primero hasta quinto grado de primaria de tres tipos lingüísticos: monolingües quechua, monolingües español y bilingües quechua-español para estudiar la formación de conceptos con el Test de Hanfman-Kasanin, diseñado en base a la teoría de Vigotski. Se apreció una superioridad de los monolingües frente a los bilingües en el nivel conceptual confirmándose la hipótesis de la interferencia entre los dos códigos lingüísticos que afectan el desarrollo cognitivo en el niño.

En los últimos años, Canales, Velarde, Meléndez y Lingán (2014 a) investigaron si existen diferencias entre habilidades psicolingüísticas evaluados con el Test de Habilidades Prelectoras, THP (Velarde, Canales Meléndez y Lingán, 2010) y los procesos de la lectura evaluados con el Prolec-R (Cuetos, 2008) en niños bilingües quechua-castellano (incipientes y avanzados) y monolingües castellano de primer grado de las regiones de Junín, Huancavelica y Lima. Encontraron que en todas las tareas psicolingüísticas evaluadas los niños bilingües incipientes rendían más bajo. 
No se encontraron diferencias significativas en los procesos de la lectura, salvo en los niños de un colegio de la muestra de Huancavelica, en la zona de Acostambo.

Canales, Velarde, Meléndez y Lingán (2014 b) realizaron otro estudio, en el que buscaban determinar si existía relación entre lenguaje oral y habilidades prelectoras, en función a la variable del Bilingüismo y la marginalidad social, en niños de 4 a 6 años de edad. Para ello se examinaron por un lado a estudiantes de educación inicial de zonas urbanas pertenecientes a Lima y Ate-Vitarte y de zonas rurales en condición de monolingües castellano en Cajamarca; y ruralesbilingües en Huancavelica. Los resultados mostraron diferencias significativas en el desarrollo lingüístico oral entre los grupos a favor de los niños de zonas urbanas, quedando en segundo término los niños de Cajamarca (Huayobamba) y en tercer lugar los de condición rural bilingüe (Quintaojos-Huancavelica) tanto en lenguaje oral como en habilidades pre lectoras.

Estudios realizados en otros países, presentan una visión distinta sobre el bilingüismo. Es el caso de Signoret (2003), quien luego de realizar una amplia revisión sobre el tema, contrastando los puntos de vista críticos, y los puntos de vista a favor de una educación bilingüe temprana, concluye que el bilinguismo brinda oportunidades y ventajas para el desarrollo cognitivo y socio-político. Calvo y Bialystok (2014) realizaron una investigación con 175 niños de 6 años de edad y que fueron distribuidos en cuatro grupos que diferían según el nivel socioeconómico y la presencia o no de una segunda lengua (monolingüe o bilingüe). Los niños fueron evaluados a través de pruebas de inteligencia no verbal, pruebas de vocabulario receptivo y de atención basada en denominación de dibujos y dos pruebas de la función ejecutiva. No se encontraron diferencias en las pruebas de inteligencia básicas, pero sí en el rendimiento en las tareas de lenguaje y función ejecutiva según el nivel socioeconómico y la presencia del bilingüismo.

En estudio reciente, Canales, Velarde, Meléndez y Lingán (2015a), estudiando las variaciones dialectales del castellano en estudiantes universitarios de educación de tres regiones del país: Lima, Huancavelica y Arequipa, empleando el Test breve de bilingüismo (Escobar 1978, González 2006) y Pruebas psicolingüísticas experimentales encontraron diferencias en la mayoría de dimensiones del lenguaje oral examinado en los estudiantes.

El caso es que cuando los niños y adolescentes ingresan al sistema educativo, traen una serie de dificultades cognitivas y psicolingüísticas- en cuya base hay una realidad neuropsicológica-, lo cual no les permite formar y adquirir las competencias básicas, indispensables para insertarse en el mundo moderno: lectura, escritura, matemáticas, ciencias. En particular es importante tomar en cuenta, no solo las dificultades en los procesos psíquicos mencionados, sino también con algunas funciones neuropsicológicas complejas que tienen que ver con la planificación, anticipación, verificación, inhibición de los impulsos y 
regulación de la conducta, funciones que según diversos estudios, corresponde ejecutar a los lóbulos frontales (Luria, 1979; Ortiz, 2004; Flores y OstroskySolis, (2008). Estudios recientes indican la relación entre nivel socio-económico y desarrollo de las funciones ejecutivas (Arán, 2011), el desarrollo de funciones ejecutivas en contextos bilingues (Martínez y Henao, 2006). También un estudio reciente nuestro: desarrollo de las funciones ejecutivas en alumnos universitarios de 3 regiones del país: Lima, Ayacucho y Huancavelica. Los últimos dos grupos, expuesto tempranamente a la condición interlectal y bilinguismo, muestran un menor desarrollo de las funciones neuropsicológicas ejecutivas (Canales, Velarde, Lingán y Echavarría, 2017).

Consideramos importante para el presente estudio, continuar la línea de investigación psicolingüística (en particular estudiando la realidad multilingüe y pluricultural del país), y articularla a los conocimientos de la neuropsicología de los lóbulos frontales y el papel que juegan en las funciones ejecutivas.

En tal sentido, en el presente estudio buscaremos analizar si existen diferencias en el desarrollo de algunas habilidades cognitivas, y funciones neuropsicológicas ejecutivas, en niños de condición bilingüe quechua-castellano y condición bilingüe shiipibo-castellano; comparados con niños monolingües castellanos; pertenecientes al mismo nivel socio-económico.

\section{Objetivo general}

Determinar si existen diferencias en los niveles de desarrollo de las habilidades cognitivas y de las funciones ejecutivas en niños bilingües y monolingües castellano, del mismo nivel socio-económico.

\section{Objetivos específicos:}

1. Evaluar y determinar el nivel de desarrollo de las habilidades cognitivas: formación de conceptos (no verbales), organización viso-espacial, capacidad de análisis y síntesis, y razonamiento.

2. Evaluar y determinar el nivel de desarrollo de las funciones neuropsicológicas ejecutivas: planeamiento, abstracción, inhibición y flexibilidad mental.

3. Comparar los niveles de rendimiento en habilidades cognitivas y funciones neuropsicológicas ejecutivas en los niños examinados de la muestra.

\section{MÉTODO}

El presente es un estudio descriptivo, de tipo causal comparativo en primer término, en donde diferenciando a los estudiantes por su realidad socio-cultural, se trata de ver como se han desarrollado las variables: inteligencia y funciones neuropsicológicas ejecutivas. 
La muestra estuvo constituida por:

- Grupo 1: 20 alumnos de $5^{\circ}$ y $6^{\circ}$ grado de la Comunidad de Socos:10 de I.E. intercultural bilingüe de Luyanta y 10 de I.E. intercultural bilingüe "Sta. Rosa de Cochabamba".

- Grupo 2: 24 alumnos de $5^{\circ}$ y $6^{\circ}$ grado de la Institución educativa de fortalecimiento de lengua originaria "Virgen del Carmen", Pilacucho, Huamanga.

- Grupo 3: 24 estudiantes de la I.E. intercultural bilingüe: "Comunidad Shipiba" en Cantagallo, Lima.

- Grupo 4: 19 estudiantes de $5^{\circ}$ y $6^{\circ}$ grado de "Santa Rosa de América" del Callao; todos ellos con edades que fluctúan entre los 10 y 12 años.

- Se controlarán las variables: edad, sexo, año de estudios, y realidad social y cultural.

\section{Técnicas e instrumentos de recolección de datos}

Se aplicaron los siguientes instrumentos:

- Prueba de matrices progresivas de Raven (test libre de cultura).

- Es un test diseñado para examinar las funciones intelectuales, a través de la detección y comparación de formas y de razonamiento analógico viso-espacial.

- Consiste en figuras geométricas que se van presentando en matrices, las cuales se van complejizando en la medida que se avanza la prueba. La resolución de dicha tarea, reflejaría el funcionamiento cognitivo del sujeto.

- Se le conoce como un test independiente de la cultura, en el sentido que no demanda mayor entrenamiento intelectual, ni recursos lingüísticos elaborados.

- Se puede aplicar en forma colectiva o individual, y en tiempo lleva unos 30 a 45 minutos realizarlo por niño.

- Para determinar su confiabilidad y validez, se trabajó con las fórmulas de Kuder-Richardson.Su confiabilidad va de 0.87 a 0.81 y su validez obtuvo un índice de 0.86(criterio de Terman Merrill).

- Pruebas de funciones ejecutivas: test de laberintos, prueba de Stroop, señalamiento autodirigido, memoria viso-espacial y Torres de Hanoi (Flores-Ostrosky, 2012). 
A propósito de la construcción del BANFE, los autores precisaron y definieron las funciones ejecutivas de la siguiente forma: Planeación: función humana primordial. Anticipación, programación, establecimiento de secuencias para la conducta que apunta a una meta. Relacionado al funcionamiento de la Corteza pre frontal dorso-lateral: CPFDL.

- Flexibilidad mental: capacidad para cambiar de plan y de acciones si no logra el resultado. Además capacidad de inhibir las anteriores respuestas. Relacionado al funcionamiento de la CPFDL.

- Control inhibitorio: en base a esta capacidad la Corteza pre frontal inhibe respuestas impulsivas, decide entre diversas respuestas posibles, inhibe patrones de respuesta cuando no son útiles. Relacionado al funcionamiento de la Corteza Orbito Frontal y Corteza Fronto Medial.

- Memoria de trabajo: es un tipo de memoria que se mantiene activa mientras el hombre está tratando de lograr sus objetivos. Baddeley (2003) la entendía como: administrador central, retén fonológico y registro visoespacial. Relacionado al funcionamiento de la CPFDorso Lateral.

- Procesamiento riesgo-beneficio: permite tomar buenas decisiones basados en estados afectivos. Participa la corteza órbito-frontal. Damasio (2001). COF y CFronto Medial.

- Abstracción: Pensamiento abstracto, clasificación lógica. CPFA.

- Metacognición: capacidad para monitorear, controlar y regular al resto de procesos cognitivos y ejecutivos.Relacionado al funcionamiento de la CPFrontal Anterior.

* Para la demostración de las Hipótesis se utilizaron técnicas de comparación de medias en grupos independientes, así como estadísticos de correlación, a un nivel de significación del 0,05

\section{RESULTADOS}

Se presentan los resultados de la investigación luego de la aplicación del Test de Matrices Progresivas para la medida de capacidad intelectual de J. C. Raven (1999) y las Pruebas neuropsicológicas de funciones ejecutivas (Flores y Ostrosky-Solis, F. 2012).

Previamente se muestran algunos detalles de la muestra.

\section{Descripción muestral}

La tabla 1 presenta los datos descriptivos de la muestra evaluada constituida por 87 alumnos. 


\section{Participantes}

Tabla 1

Características de la muestra

\begin{tabular}{lcccc}
\hline & Socos & Huamanga & Cantagallo & Callao \\
\hline $\mathrm{N}$ & 20 & 24 & 24 & 19 \\
Género & & & & \\
$\quad$ Masculino & $9(45 \%)$ & $14(58.3 \%)$ & $14(58.3 \%)$ & $14(73.7 \%)$ \\
$\quad$ Femenino & $11(55 \%)$ & $10(41.7 \%)$ & $10(41.7 \%)$ & $5(26.3 \%)$ \\
Grado escolar & & & & \\
$\quad$ 5to. grado & $20(100 \%)$ & $14(58.3 \%)$ & $12(50 \%)$ & $10(52.6 \%)$ \\
$\quad$ 6to. grado & $0(0 \%)$ & $10(41.7 \%)$ & $12(50 \%)$ & $19(47.4 \%)$ \\
Prom. de edad (DE) & $10.60(.681)$ & $10.83(.637)$ & $10.88(.741)$ & $10.94(.680)$ \\
\hline
\end{tabular}

En el presente estudio, se examinó a 4 grupos de alumnos de 5to. Y 6to. de primaria: 20 alumnos de la Comunidad de Socos (Ayacucho) que asisten a una Escuela Intercultural Bilingüe, que reciben educación en castellano y parcialmente en quechua pero con entorno cultural y lingüístico de alta influencia quechua, con promedio de edad: 10.6 años; 24 alumnos de una Institución Educativa de Pilacucho, Huamanga (Ayacucho) con educación en castellano, con grado menor de influencia quecha, con promedio de edad: 10.83 años; 24 alumnos de la comunidad Shipiba asentados en Cantagallo, distrito del Rímac en Lima que reciben educación en castellano y parcialmente en su lengua originaria, pero con entorno cultural y lingüístico de alta influencia del castellano,con promedio de edad: 10.88 años; y por último, 19 alumnos hispanohablantes de una escuela pública del Cercado del Callao, perteneciente a la Provincia Constitucional del Callao con promedio de edad: 10.94 años.

Se pudo apreciar que la zona y tipo de viviendas en el caso de la Comunidad de Socos, era de tipo rural. A diferencia de los grupos de Huamanga, Lima (Cantagallo) y Callao, que es de tipo urbano, y en tal sentido estos últimos cuentan mayoritariamente con acceso a los servicios básicos: agua, desagüe, teléfono, cable, internet.

De otro lado, respecto a aspectos culturales y lingüísticos, se aprecia que hay una gradiente marcada acerca de la lengua materna predominante: mayoritariamente el quechua en Socos, luego quechua-castellano en Huamanga, castellano y shipibo en Cantagallo (Lima) y castellano en el Callao.

\section{Verificación de Hipótesis}

Haciendo la comprobación de HIPÓTESIS, se tiene que: 
- H1: Existen diferencias en el nivel de desarrollo de las habilidades cognitivas en los alumnos de la muestra considerando su realidad psicolingüística: bilingües (lengua nativa-castellano) y monolingües castellano.

Diferencias en el desempeño del Test de Raven

Tabla 2

Comparación de grupos según los resultados obtenidos en el Test de Raven (razonamiento perceptivo)

\begin{tabular}{|c|c|c|c|c|c|c|}
\hline & \multicolumn{4}{|c|}{ Rango promedio } & \multirow{2}{*}{$\mathrm{X}^{2 \mathrm{a}}$} & \multirow{2}{*}{$\mathbf{p}^{\mathbf{a}}$} \\
\hline & Socos & Huamanga & Cantagallo & Callao & & \\
\hline Escala A & 27.30 & 44.98 & 56.33 & 42.42 & 15.822 & .001 \\
\hline Escala Ab & 23.78 & 34.04 & 62.88 & 52.19 & 33.153 & .000 \\
\hline Escala B & 27.50 & 41.44 & 56.38 & 46.86 & 15.469 & .001 \\
\hline Raven & 22.05 & 38.06 & 62.56 & 49.17 & 30.957 & .000 \\
\hline
\end{tabular}

aSegún la prueba de Kruskal-Wallis (grados de libertad: 3 )

En la tabla 2 se aprecia que se encontraron diferencias significativas entre los grupos de estudiantes de diferentes realidades sociolingüísticas en su desempeño en las escalas y el puntaje total del Test de Raven. Al hacer un análisis de pares, no paramétrico, con el estadístico de Mann Whitney se encontró que las diferencias en la puntuación total se presentan entre: los estudiantes de Socos y cada uno de los otros grupos de estudiantes (Huamanga: $\mathrm{U}=114.000, \mathrm{p}=.003$; Cantagallo: $\mathrm{U}$ $=54.000, \mathrm{p}=.000$; Callao: $\mathrm{U}=63.000, \mathrm{p}=.000$ ); en todos los casos, los niños de Socos obtienen puntuaciones inferiores que sus pares.

De otro lado, los estudiantes de Cantagallo obtienen puntuaciones que superan a los estudiantes de Huamanga $(\mathrm{U}=105.000, \mathrm{p}=.000)$ y a los estudiantes de Callao $(\mathrm{U}=127.500, \mathrm{p}=.024)$.

Resultados similares se encuentran en las escalas A (Huamanga: $U=138.000$, $\mathrm{p}=.014$; Cantagallo: $\mathrm{U}=84.000, \mathrm{p}=.000$ ), Ab (Huamanga: $\mathrm{U}=155.000, \mathrm{p}=.042$; Cantagallo: $U=54.000, p=.000$; Callao: $U=56.500, p=.000$ ) y B (Huamanga: $U$ $=148.000, p=.027$; Cantagallo: $U=93.000, p=.000$; Callao: $U=99.000, p=.017$ ) del Raven, en los que los niños de Socos obtienen puntuaciones significativamente inferiores que sus pares. Sus puntuaciones solo se equiparan a las de los estudiantes del Callao en la escala A $(\mathrm{U}=114.000, \mathrm{p}=.055)$. Entre los niños de Huamanga y Callao, solo se encuentran diferencias en la escala $\mathrm{Ab}(\mathrm{U}=92.500, \mathrm{p}=.060)$, a favor del grupo del Callao. 
De modo general, se puede afirmar que existen diferencias significativas en el rendimiento cognitivo, entre los grupos de realidad socio-cultural y lingüística bilingüe quechua-castellano del interior del país, comparados con los grupos predominantemente castellano-hablantes de la capital, a favor de estos últimos.

Particularmente se debe señalar que comparando los grupos de realidad socio-cultural de la capital, el grupo de niños shipibo-castellano presenta un mejor rendimiento.

- H2: Existen diferencias en el nivel de desarrollo de las funciones neuropsicológicas ejecutivas en los alumnos de la muestra considerando su realidad psicolingüística: bilingües (lengua nativa-castellano) y monolingües castellano.

\section{Diferencias en el desempeño del Banfe}

Tabla 3

Comparación de grupos según los resultados obtenidos en las tareas del BANFE

\begin{tabular}{lccccccc}
\hline & \multicolumn{5}{c}{ Rango promedio } & \multirow{2}{*}{$\mathbf{X}^{2 \mathbf{a}}$} & \multirow{2}{*}{$\mathbf{p}^{\mathbf{a}}$} \\
\cline { 2 - 4 } & Socos & Huamanga & Cantagallo & Callao & & \\
\hline -SubtestOrbitomedial & 29.43 & 34.38 & 56.67 & 52.11 & $\mathbf{1 8 . 5 2 7}$ & $\mathbf{. 0 0 0}$ \\
$\begin{array}{l}\text {-SubtestDorsolateral } \\
\text { Memoria de trabajo }\end{array}$ & 43.08 & 53.96 & 45.26 & 25.42 & $\mathbf{1 4 . 1 5 7}$ & $\mathbf{. 0 0 3}$ \\
$\begin{array}{l}\text {-SubtestDorsolateral } \\
\text { Funciones ejecutivas }\end{array}$ & 26.25 & 45.52 & 55.17 & 42.69 & $\mathbf{1 5 . 0 7 5}$ & $\mathbf{. 0 0 2}$ \\
-SubtestDorsolateral & 27.58 & 48.17 & 56.39 & 36.14 & $\mathbf{1 7 . 0 3 9}$ & $\mathbf{. 0 0 1}$ \\
\hline
\end{tabular}

aSegún la prueba de Kruskal-Wallis (grados de libertad: 3)

De acuerdo a la tabla 3, se encontraron diferencias significativas entre los grupos de estudiantes de diferentes realidades sociolingüísticas en su desempeño en las tareas que componen el BANFE. Al hacer un análisis de pares, no paramétrico, con el estadístico de Mann Whitney se encontró que las diferencias en el subtestorbitomedial se presentan entre: los estudiantes de Socos y los de Cantagallo ( $\mathrm{U}=92.500, \mathrm{p}=.001)$, y entre los estudiantes de Socos y los de Callao $(\mathrm{U}=73.500, \mathrm{p}=.001)$, en ambos casos las puntuaciones inferiores corresponden a los niños de Socos. Así mismo, también se halló que los niños de Huamanga obtienen puntuaciones estadísticamente inferiores en comparación con sus pares de Cantagallo $(\mathrm{U}=140.000, \mathrm{p}=.004)$ y Callao $(\mathrm{U}=117.500, \mathrm{p}=.012)$.

En el subtestdorsolateral de memoria de trabajo, llama la atención que los niños de Callao presenten resultados estadísticamente inferiores que los estudiantes de Socos $(U=100.500, p=.019)$, los de Huamanga $(U=78.500, p=.000)$ y los de 
Cantagallo $(\mathrm{U}=107.500, \mathrm{p}=.009)$. Estos resultados contrastan con lo hallado en el subtestdorsolateral de funciones ejecutivas, siendo los niños de Socos los que obtienen las puntuaciones significativamente inferiores en comparación con sus pares de Huamanga $(\mathrm{U}=123.500, \mathrm{p}=.006)$, de Cantagallo $(\mathrm{U}=92.000, \mathrm{p}=.001)$ y de Callao $(U=99.500, p=.017)$. No se analizan los resultados encontrados en la sumatoria de las puntuaciones de los subtestsdorsolateral debido a la falta de evidencias para corroborar la validez de la sumatoria como constructo unitario.

En términos generales se puede afirmar que existen diferencias significativas entre el rendimiento en funciones neuropsicológicas ejecutivas, de los grupos de realidad socio-cultural y lingüística bilingüe quechua-castellano del interior del país, comparados con los grupos predominantemente castellano-hablantes de la capital, a favor de estos últimos, siendo los de más bajo rendimiento los niños de Socos.

Efectivamente llama la atención, que el grupo del Callao, haya rendido tan bajo en la prueba neuropsicológica correspondiente a las funciones ejecutivas de memoria de trabajo, siendo superado por los niños de Socos y Huamanga. Los niños del grupo bilingüe shipibo-castellano que vive en la capital, también rindió en las pruebas neuropsicológicas mejor que los otros grupos, a excepción de la prueba de memoria de trabajo.

\section{Hipótesis complementaria:}

- Existe asociación entre las habilidades cognitivas examinadas y las funciones neuropsicológicas ejecutivas en la muestra estudiada.

Correlaciones entre habilidades cognitivas y funciones ejecutivas

Tabla 4

Correlaciones entre las escalas del RAVEN y las funciones ejecutivas

\begin{tabular}{lcccc}
\hline & $\begin{array}{c}\text { Subtest } \\
\text { Orbitomedial }\end{array}$ & $\begin{array}{c}\text { SubtestDorsolateral } \\
\text { Memoria de trabajo }\end{array}$ & $\begin{array}{c}\text { SubtestDorsolateral } \\
\text { Funciones ejecutivas }\end{array}$ & $\begin{array}{c}\text { Sumatoria } \\
\text { Dorsolateral }\end{array}$ \\
\hline Escala A & .189 & -.014 & $\mathbf{. 3 1 1 * *}$ & $.287^{* *}$ \\
Escala Ab & $\mathbf{. 3 1 6 * *}$ & -.119 & $\mathbf{. 3 4 0 * *}$ & $.298^{* *}$ \\
Escala B & $\mathbf{. 3 3 9 * *}$ & -.181 & $\mathbf{. 3 2 2 * *}$ & $.250^{*}$ \\
Raven & $\mathbf{. 3 6 3 * *}$ & -.156 & $\mathbf{. 4 0 1 * *}$ & $.336^{* *}$ \\
\hline
\end{tabular}

Nota: Se muestran los coeficientes rho de Spearman; $\mathrm{N}=85$.

$* \mathrm{p}<.05 ; * * \mathrm{p}<.01$

En la tabla 4 se aprecian los coeficientes de correlación rho de Spearman, encontrándose correlación estadísticamente significativa entre las puntuaciones del subtestorbitomedial y las escalas Ab y B del Raven; así mismo, todas las puntuaciones de las escalas del Raven correlacionaron significativamente con las obtenidas en el subtestdorsolateral de funciones ejecutivas. Es importante destacar que no se hallaron correlaciones significativas entre las respuestas de las escalas 
del Raven y las del subtestdorsolateral de memoria de trabajo, por tal motivo, no se resaltan los coeficientes de correlaciones significativos hallados con las sumatorias de los subtestsdorsolateral. Esto cuestiona la validez de asumir una sumatoria entre ambas tareas: memoria de trabajo y funciones ejecutivas.

Analizando los resultados en términos globales, se afirma que existen diferencias significativas entre los grupos, tanto en funcionamiento cognitivo: inteligencia, razonamiento espacial; asi como en las funciones ejecutivas: flexibilidad mental, abstracción, planificación e inhibición de impulsos, que dependen de áreas del lóbulo frontal.

Quienes rindieron más bajo en ambas variables (a excepción de la función ejecutiva: memoria de trabajo) fueron los niños de Socos, Ayacucho, zona rural y quechua-hablante. Rindieron mejor los alumnos de Huamanga, capital del departamento, que tanto a nivel familiar como escolar, están expuestos a la condición interlectal, predominantemente castellano, con cierta presencia del quechua.

Finalmente, fueron los alumnos del Callao, hispano hablantes; y los niños de la comunidad shipiba de Cantagallo, ambos grupos de la capital del país, los que rindieron mejor en ambas variables (a excepción del bajo rendimiento en la prueba neuropsicológica de memoria de trabajo del grupo del Callao).

Respecto a las correlaciones entre funciones cognitivas y las funciones neuropsicológicas ejecutivas, se hallaron correlaciones estadísticamente significativas entre los rendimientos en las diversas escalas del test de Raven, y los subtests relacionados al funcionamiento ejecutivo que evalúa la prueba BANFE, a excepción del subtest que examina el funcionamiento del área dorso-lateral Memoria de trabajo.

\section{DISCUSIÓN}

En relación a las funciones cognitivas y neuropsicológicas ejecutivas apreciamos que aparecen diferencias significativas entre los grupos, en este caso a favor de los grupos de Lima: grupo de la comunidad shipiba que recibe educación principalmente en castellano (con cierta influencia de bilingüismo shipibocastellano) y el grupo castellano hablante del Callao.

Este resultado, de algún modo, respaldaría la importancia que tienen los factores disortogénicos (González, 2006): pobreza extrema, marginación social, analfabetismo de los padres, el problema del bilingüismo quechua-castellano, estarían relacionados con el desarrollo cognitivo y de funciones ejecutivas en los niños. Ello estaría operando con más fuerza en las poblaciones del interior del país, especialmente en las zonas rurales con predominancia de lengua materna quechua y con niños expuestos tempranamente al bilingüismo quechua-castellano. 
Es importante señalar, que precisamente por ello, es que se habla de bilingüismo sustractivo en el Perú, en tanto el factor lingüístico no actúa como factor aislado, sino articulado a los otros factores.

En tal sentido, y tal como González (2006) y Canales et al (2014b) lo señalan, las comunidades que presentan doble marginalidad: socio-económica y lingüística, se verían más perjudicadas en su desarrollo psicológico, que aquellas que presentan una sola marginalidad (económica o lingüística).

Analizando un punto importante en los resultados: la diferencia entre los 2 grupos de Lima, a favor del grupo de los niños shipibos, ello podría explicarse tentativamente del modo siguiente: A) Los shipibos se encuentran en Lima, la capital, hace 15 años, cuando llegaron huyendo de la violencia y buscando mejores horizontes para sus familias. Con el tiempo se fueron asentando, la mayoría se dedicó al comercio de artesanías y otros, y desde esa posición se relacionaron con los habitantes de la capital. En tal sentido se les puede considerar un sector social emergente. B) La asimilación y estructuración de la lengua 2 que ellos han ido realizando se puede considerar como de un buen nivel. No solo los niños usan y manejan el castellano de buen modo, sino también sus padres y familiares. C) Los shipibos se pueden considerar una comunidad relativamente cerrada que cultiva sus tradiciones, cultura, lengua, en forma más ordenada, sistemática, con una escuela intercultural mejor organizada, en comparación a los niños de escuelas de la zona rural de Ayacucho, o de los propios niños del Callao, que tienen, aparte de escuelas con maestros no muy actualizados y preparados, un contexto social y familiar más anómico, con crisis de familias, etc.

Un resultado parecido encontramos en el estudio de Canales et al (2014a) en donde apreciamos que los niños de Acostambo, Huancavelica, rendían mejor que los niños de Vitarte (Huaycán) en lectura al finalizar el año escolar. Ahí interpretamos que otros factores también operaban en el buen desarrollo cognitivo y de aprendizaje, tal como era la Gestión y la organización escolar.

Finalmente, se puede afirmar que en términos de bilingüismo la comunidad shipiba de Lima, podría estar desarrollando un bilingüismo coordinado, es decir el de las personas que diferencian las estructuras internas y la conducta lingüística externa de las 2 lenguas. También sería un bilingüismo temprano (antes de los 4 años fueron ingresados al castellano) y bilingüismo estable, porque son grupos migrantes que mantienen su lengua originaria (aunque de menor uso en la capital), tienen conciencia e identidad de grupo, y buscan mantener, fortalecer y promover en sus hijos su lengua nativa (con cierto orgullo cultural, lingüístico e incluso étnico) lo cual no se aprecia en las poblaciones quechuas de nuestra sierra central acaso por todos los sufrimientos, violencia, marginación y desprecio del que han sido objeto históricamente. 
Este resultado general nos lleva a pensar, que a diferencia de lo que planteaban otros investigadores (Siguan, 1996, Signoret, 2003) que señalan que el bilingüismo brinda ventajas para el desarrollo cognitivo, en el Perú el asunto es completamente diferente. Lo que ocurre aquí respecto al bilingüismo quechua-castellano es distinto. Nuestro bilingüismo tiene carácter de incompleto, desigual y sustractivo (González, 2006; Canales, Velarde, Meléndez y Lingán 2015).

Y es precisamente, la falta de una visión objetiva y un tratamiento adecuado a la condición bilingüe del estudiante, especialmente de las zonas alto andinas, lo que no permite su avance hacia mayores niveles de funcionamiento cognitivo y neuropsicológico, base para lograr buenos procesos de aprendizaje y un buen desarrollo psicológico, todo lo cual repercuta en su inserción e integración social y su futuro como ciudadano de la república.

\section{CONCLUSIONES}

En relación a las funciones cognitivas y las funciones neuropsicológicas ejecutivas, se aprecia que aparecen diferencias significativas entre los grupos, a favor de los grupos de Lima, grupo del Callao) y especialmente, a favor del grupo de la comunidad shipiba en Lima.

Los factores disortogénicos (González, 2006): pobreza extrema, marginación social, analfabetismo de los padres, el problema del bilingüismo quechuacastellano, todos ellos juntos, tal como se da en las zonas alto andinas, estarían relacionados con el desarrollo cognitivo y de funciones ejecutivas en los niños.

Respecto al buen rendimiento de los niños de la comunidad shipiba de Cantagallo, Lima, se interpreta que ello se debería a tres condiciones: a) Los shipibos entendidos como sector emergente. B) La buena asimilación y estructuración de la lengua 2: el castellano. C) Los shipibos como una comunidad relativamente cerrada que cultiva sus tradiciones, cultura, lengua, en forma más ordenada, sistemática.

Los instrumentos aplicados demostraron ser válidos y útiles para el examen de las habilidades cognitivas, la inteligencia y las funciones ejecutivas neuropsicológicas.

\section{RECOMENDACIONES}

- Tomar en cuenta que el desarrollo cognitivo y la organización de las funciones neuropsicológicas cerebrales, están asociadas a los factores disortogénicos (presente en las zonas rurales del interior, especialmente andinas) junto a la condición del bilinguismo en nuestro país. 
- Sobre la base de estos resultados, tratar de avanzar al diseño de programas de intervención para reducir dichos efectos disortogénicos en nuestros niños.

- Finalmente, también de acuerdo a nuestros resultados, ingresar al debate nacional, tratando de caracterizar de mejor modo, los problemas más acuciantes del país, y de cómo hasta el momento no se han podido superar, y aportar de mejor modo en el diseño de políticas educativas que apunten no solo a la calidad, sino también a la equidad, base para un verdadero desarrollo del país.

\section{REFERENCIAS BIBLIOGRÁFICAS.}

Arán, V. (2011). Funciones ejecutivas en niños escolarizados: efectos de la edad y del estrato socioeconómico. Avances en Psicología Latinoamericana/Bogotá (Colombia)/Vol. 29(1)/pp. 98-113

Baddeley, A. (2003). Working Memory. Looking back and looking forward. Nature Review Neuroscience, 4 (10). 829-839

Calvo, A. y Bialystok, E. (2014). Independent Effects of Bilingualism and Socioeconomic Status on Language Ability and Executive Functioning. Cognition. 2014 Mar; 130(3): 278-288.

Canales R., Velarde E., Meléndez M., Lingán S (2014a). Diferencias en Habilidades psicolingüísticas y lectura en niños bilingües quechua-castellano y monolingües castellano de primer grado. En Theorema, Revista del Vicerrectorado de Investigación UNMSM. Vol I N¹. Segunda época, junio 2014.

Canales R., Velarde E., Meléndez M., Lingán S (2014b) Lenguaje oral y habilidades prelectoras en niños de 4 a 6 años. Un estudio sobre marginalidad y bilingüismo en el Perú. Revista de Investigación en Psicología. IIPSI. Facultad de Psicología, UNMSM. Vol.17-N¹-2014, pp, 107-119.

Canales R., Velarde E., Meléndez M., Lingán S. (2015). "Variaciones dialectales del castellano y distancias comunicacionales en estudiantes universitarios iniciales de educación. Un estudio sobre habilidades psicolingüísticas y bilingüismo en el Perú". Revista de Investigación en Psicología. IIPSI. Facultad de Psicología, UNMSM. Vol.18-N²-2015, pp, 71-82.Lima. UNMSM.

Canales R., Velarde E., Lingán S. y Echavarría, L. (2017) “Funciones ejecutivas y Juicio moral en alumnos pertenecientes realidades socio-culturales distintas: Lima, Ayacucho y Huancavelica". Revista de Investigación en Psicología. IIPSI. Facultad de Psicología, UNMSM. Vol.20-Nº1-2017, pp, 47-66. Lima. UNMSM.

Cuetos, F. (2008). Psicología de la lectura. Educación infantil y primaria. España. Ediciones Wolters Kluwer.

Damasio, A. (2001). El error de Descartes. España. Destino.

Escobar, A. (1978). Variaciones sociolingüísticas del castellano en el Perú. Lima. Instituto de Estudios Peruanos. 
Flores, J.C. y Ostrosky-Solis, F. (2008). Batería de funciones ejecutivas, presentación. Revista Neuropsicología, Neuropsiquiatría y Neurociencias 8 (1). 151-158

Flores, J. C. y Ostrosky-Solis, F. (2012). Desarrollo neuropsicológico de lóbulos frontales y funciones ejecutivas. México. Manual Moderno.

González, R. (2006). Problemas psicolingüísticos en el Perú. Obras Completas. Volumen I. Lima: Ediciones. N. Reátegui.

Martinez, M. y Henao, G. (2006). Desempeño en la función ejecutiva en niños expuestos a ambientes de enseñanza bilingüe. Revista electrónica de investigación psicoeducativa. $\mathrm{N}^{\circ}$ 10. Vol. 4 (3). 513-528

Luria, A. (1979). El cerebro en acción. Barcelona. Edit. Fontanella.

Ortiz, P. (2004). Introducción a la psicobiología del hombre. Lima. Fondo editorial UNMSM.

Raven, J. C. (1999). Test de Matrices Progresivas. Buenos Aires. Paidos.

Siguan, M. (coord.) (1996). La enseñanza precoz de una segunda lengua en la escuela, Barcelona, Horsoni.

Signoret, A. (2003). Bilingüismo y cognición: ¿cuándo iniciar el bilingüismo en el aula? Perfiles Educativos, vol. XXV, núm. 102, Instituto de Investigaciones sobre la Universidad y la Educación, México.

Velarde, E., Canales, R., Meléndez, M. y Lingán, S. (2010). Enfoque cognitivo y psicolingüístico de la lectura: diseño y validación de una prueba de habilidades prelectoras (THP) en niños y niñas de la Provincia Constitucional del Callao, Perú. Revista de Investigación en Psicología. IIPSI. Facultad de Psicología. Vol.13-N¹-2010, pp, 53-68.Lima. UNMSM. 